\title{
Incidental detection of ATTR cardiac amyloidosis
}

\author{
Jessica A. Quaggin-Smith, ${ }^{\text {a Ramsey } M . \text { Wehbe, }^{\mathrm{b}} \text { and Thomas A. Holly }}{ }^{\mathrm{b}}$ \\ a Department of Medicine, Northwestern University Feinberg School of Medicine, Chicago \\ b Division of Cardiology, Northwestern University Feinberg School of Medicine, Chicago
}

Received Nov 20, 2020; accepted Nov 20, 2020

doi: $10.1007 / \mathrm{s} 12350-020-02467-9$

\section{See related article, pp. $1021-1029$}

Despite increased recognition of the disease and new treatment options, transthyretin cardiac amyloidosis (ATTR-CA) remains an underdiagnosed cause of heart failure $^{1-3}$. ATTR-CA is a subtype of systemic amyloidosis caused by misfolding of either a wild-type or mutated transthyretin protein and deposition of extracellular ATTR amyloid fibrils in myocardial tissues leading to heart failure and arrhythmias, typically in an older population ${ }^{1,3}$.

Once thought to be a rare disease, it has been estimated that $25 \%-36 \%$ of the population over 80 are at risk for developing symptomatic wild-type cardiac amyloidosis. ${ }^{1}$ In fact, in one study, examining autopsy results of older ( $>80$ years old) patients, $25 \%$ were found to have wild-type cardiac amyloid. ${ }^{2}$ This figure is not including hereditary ATTR-CA, the prevalence of which varies depending on the mutation ${ }^{2,4,5}$. Unfortunately, the disease also carries a high mortality rate without treatment, it is estimated that mean survival for ATTR-CA patients with heart failure is $\sim 2.5-3.5$ years after diagnosis ${ }^{6}$. Standard heart failure therapies, such as an ICD, have not been shown to clearly benefit patients with ATTR-CA ${ }^{7,8}$. Similarly, many amyloid patients struggle to tolerate current guideline-directed medical therapies for heart failure and some options, such as beta-blockers, can actually worsen outcomes for patients through their negative inotropic and chronotropic

Reprint requests: Thomas A. Holly, Division of Cardiology, Northwestern University Feinberg School of Medicine, Chicago; tholly@nm.org

J Nucl Cardiol 2022;29:1030-3.

$1071-3581 / \$ 34.00$

Copyright (C) 2021 American Society of Nuclear Cardiology. effects. ${ }^{7}$ Aside from solid organ transplantation, the only effective treatment that is currently FDA-approved, the transthyretin stabilizer tafamadis, works by preventing further accumulation of the misfolded protein in cardiac tissues. While this treatment offers a remarkable survival benefit, this benefit was only seen starting approximately 18 months after initiation of therapy. ${ }^{9}$ This suggests that treatment may work by slowing disease progression but is unable to reverse the damage that has already been caused by the amyloid fibrils. ${ }^{9}$ Thus, early diagnosis is key to improve outcomes for patients.

Even with increased recognition of ATTR-CA disease prevalence, which disproportionately falls on the elderly, diagnosis has remained challenging. ${ }^{10}$ Endomyocardial biopsy is the reference standard for diagnosis, but is invasive, associated with non-negligible risk of complications, and is not a readily accessible procedure to much of the population as it requires particular expertise and resources typically available only at large academic centers. Non-invasive diagnostic studies including electrocardiography (ECG), echocardiography, and cardiac MRI can be helpful in suggesting a diagnosis of ATTR-CA, ${ }^{10,11}$ but cardiac scintigraphy with bone-avid tracers has emerged as a key diagnostic test in establishing a non-invasive diagnosis of ATTRCA.

Cardiac scintigraphy for ATTR-CA relies on the principle that calcium-avid radiotracers, including 99mTc-labeled pyrophosphate ( ${ }^{99 \mathrm{~m}}$ Tc-PYP), 3,3-diphosphono-1,2-propanodicarboxylic acid ( $\left.{ }^{99 \mathrm{~m}} \mathrm{Tc}-\mathrm{DPD}\right)$, or hydroxymethylene diphosphonate $\left({ }^{99 \mathrm{~m}} \mathrm{Tc}-\mathrm{HMDP}\right)$, are taken up by the myocardium preferentially in ATTR-CA due to extracellular calcium associated with ATTR amyloid fibrils. Images are typically obtained 1 hour post injection. In some cases repeat images are obtained 3 hours post injection. Interpretation involves semiquantitative analysis with the Perugini grade in which myocardial uptake is compared to rib uptake (with grade 2 or 3 uptake considered positive for ATTR-CA ${ }^{12}$ ) and 
quantitative analysis with the heart/contralateral lung (H/CL) ratio of counts (with higher ratios indicating higher tracer uptake in the myocardium ${ }^{13}$ ). Combined with a laboratory evaluation to exclude a diagnosis of amyloid light-chain (AL) amyloidosis, cardiac scintigraphy can be used to establish a non-invasive diagnosis of ATTR-CA with prior studies quoting sensitivities and specificities of $97 \%$ and $100 \%$, respectively. ${ }^{13,14}$ Cardiac scintigraphy has also been shown to detect ATTR-CA earlier than other testing modalities, with positive uptake noted on scans in patients with normal echocardiograms and ECGs. ${ }^{15}$ This is thought to be due to tracer uptake representing degree of amyloid infiltration, with decreased quantitative and qualitative uptake representing a lower degree of infiltration. ${ }^{15}$ This represents an opportunity to capture the disease in its earlier stages before cardiac dysfunction appears. Given its many positive attributes, it has been suggested that cardiac scintigraphy could represent a new non-invasive "gold standard" for diagnosis, although both pre-test probability of ATTR-CA in a population as well as reader/ technician experience remain important considerations in the utility of this test. ${ }^{16}$

Given the importance of early diagnosis of ATTRCA and the potential of cardiac scintigraphy to identify subclinical disease, in this issue of the Journal of Nuclear Cardiology Suomalainen et al. ${ }^{7}$ explored whether there was prognostic significance to incidentally found suspected ATTR-CA on bone scintigraphy studies performed for other reasons. This study evaluated 2000 patients over the age of 70 and compared the risk of overall and cardiovascular mortality between patients with positive ${ }^{99 \mathrm{~m}}$ Tc-HMDP scans (grade 2 uptake or $\mathrm{H} /$ $\mathrm{CL}$ ratio $>1.30$ at 3 hours) and those with negative ones. Of the study's patient population, 69 (3.5\%) were suspected to have ATTR-CA based on these scans (only $41 \%$ of whom had an established diagnosis of heart failure at the time of imaging). The investigators found that those with grade 3 uptake had an increased risk of both overall and cardiovascular mortality, while those with grade 2 had an increased risk of cardiovascular mortality only. A higher H/CL ratio was also associated with both increased cardiovascular and all-cause mortality. It is important to note that diagnosis was based off imaging and there were no biopsy-proven cases, making it difficult to say how many of the study patients truly have ATTR-CA according to current diagnostic guidelines.

This study, in part, solidifies what we already know - that cardiac uptake on bone scintigraphy imaging is associated with an increased risk for ATTR-CA and worse clinical prognosis. However, findings from this study raise important questions that are worth exploring further. First, while findings on routine bone scintigraphy suggestive of ATTR-CA appear to be associated with an increased risk of morbidity and mortality, it is yet unclear whether targeting therapy to this population could change outcomes. As mentioned earlier, currently available therapies rely on stabilizing the transthyretin protein. ${ }^{9}$ Newer drugs within ongoing clinical trials, such as doxycycline and anti-sense oligonucleotides, also rely on stopping progression of the disease rather than disease reversal. ${ }^{7}$ This makes a population with incidentally noted disease, only $41 \%$ of whom have established heart failure, a potentially exciting population with mostly subclinical disease in which targeted therapies might be more effective when started earlier in their disease course.

However, one should be cautious in drawing conclusions about the effectiveness of therapy in these patients, given the comorbidities in a population referred for bone scintigraphy. Almost all of the patients in this study were referred to detect the presence of bone metastases in the setting of primarily breast or prostate cancer. In contrast, only 29 patients $(11 \%)$ had coexistent cancer in the landmark ATTR-ACT trial which established the effectiveness of tafamadis in ATTRCA. ${ }^{9}$ Therefore, an important consideration is the competing risk of death from malignancy. Interestingly, in the current study by Suomalainen et al., while patients with grade 3 uptake had higher overall and cardiovascular mortality even after controlling for metastatic disease, patients with grade 2 uptake had no difference in overall mortality compared to the patients without ATTR-CA despite an increased risk of cardiovascular mortality. This is contrary to prior studies in which there was no reported significant difference in outcomes between patients with grade 2 and grade 3 uptake $^{17,18}$ and could be explained by the complex interplay of factors related to the comorbidity of cancer. For example, the competing risk of death from cancer might be higher than the risk of death from ATTR-CA in those patients with a lower burden of cardiac infiltrative disease on imaging or the competitive uptake of radionuclide from bony metastases may lead to less prominent cardiac uptake in patients with a higher burden of metastatic disease. Either way, the question remains if these patients' trajectories can be changed with current treatment regimens and, in the same vein, if they would gain any benefit from incidental detection. Even if we are unable to provide an overall mortality benefit with our available medical therapies, it is possible that we can offer an increased quality of life for these patients by preventing the development of significant heart failure symptoms.

Finally, there are questions as to the cost-effectiveness of a strategy of treating patients with early, subclinical ATTR-CA even if treatment was found to be 
clinically effective in these patients. Even in patients with established heart failure symptoms and cardiomyopathy due to ATTR-CA, at current drug prices in the United States, tafamadis was not found to be cost-effective. ${ }^{19}$ Screening for ATTR-CA routinely on bone scintigraphy performed for other causes would be very likely to increase the population with prevalent disease, and therefore, further exacerbate the financial burden of treatment. Significant price reductions in available therapies for ATTR-CA would likely be necessary to make early treatment more cost-effective and increase the adoption of a strategy of screening for incidental disease on routine bone scintigraphy.

Looking more broadly, if screening were to be implemented in patients undergoing bone scintigraphy for non-cardiac causes, there are a few questions that still need to be answered. First, it is unclear how well a protocol that is not designed nor previously validated for the detection of ATTR-CA (3 hour imaging only, lack of consistent SPECT imaging) performs in establishing a diagnosis compared to dedicated cardiac scintigraphy protocols. Another key consideration is the interpreting radiologist or nuclear medicine specialist. In order to appropriately screen patients for cardiac amyloid, all physicians reading these scans would need to know to specifically look for cardiac amyloid, as well as how to appropriately grade these scans. They should also understand that there are pitfalls in the interpretation of this form of imaging that can generate false positive and false negative results, such as recent myocardial infarction, AL cardiac amyloidosis, delayed blood pool clearance from renal dysfunction and dilated cardiomyopathy, and focal intra/extracardiac uptake. ${ }^{20}$ In addition, H/CL ratio should be calculated on these studies, at least those with grade 2 or 3 uptake. This would require establishing screening guidelines for patients receiving these scans for non-cardiac diagnoses, as well as ensuring appropriate referral to a cardiologist if the scan is read as concerning for ATTR-CA. This would help ensure that institutions and departments are appropriately offering this service to patients. Finally, patients should be offered the opportunity for shared decision-making and be informed that even with a true positive study it is not clear that current therapies for ATTR-CA would alter an oncology patient's clinical prognosis.

In all, the report by Suomalainen et al. raises important questions about the utility of screening patients for ATTR-CA, particularly those without heart failure symptoms. It is clear that with current and emerging therapies, there is now enormous interest in earlier identification of ATTR-CA to shift the paradigm from treatment to prevention of cardiac disease. Cardiac scintigraphy appears to be a compelling tool for this purpose. However, many questions remain as to the appropriate population to screen to target therapy where it will be most clinically and cost-effective. This serves as a call to action, as research in this space is desperately needed. As our technology and understanding advances, especially given a number of ongoing clinical trials for novel drug therapies, screening the appropriate patient population for ATTR-CA will likely result in meaningful improvements in patient outcomes and quality of life.

\section{References}

1. Liao R, Ward JE (2017) Amyloid cardiomyopathy - disease on the rise. Circ Res 120:1865-1867

2. Mankad AK, Shah KB (2017) Transthyretin cardiac amyloidosis. Curr Cardiol Rep 19:97

3. Cornwell GG, Murdoch WL, Kyle RA, Westermark P, Pitkänen P (1983) Frequency and distribution of senile cardiovascular amyloid: A clinicopathologic correlation. Am J Med 75:618-623

4. Jacobson DR, Alexander AA, Tagoe C, Buxbaum JN (2015) Prevalence of the amyloidogenic transthyretin (TTR) V122I allele in 14333 African-Americans. Amyloid 22:171-174

5. Buxbaum J, Jacobson DR, Tagoe C et al (2006) Transthyretin V122I in African Americans with congestive heart failure. J Am Coll Cardiol 47:1724-1725

6. Ruberg FL, Grogan M, Hanna M, Kelly JW, Maurer MS (2019) Transthyretin amyloid cardiomyopathy: JACC state-of-the-art review. J Am Coll Cardiol 73:2872-2891

7. Suomalainen O, Pilv J, Loimaala A, Mätzke S, Heliö T, Uusitalo V (2020) Prognostic significance of incidental suspected transthyretin amyloidosis on routine bone scintigraphy. J Nucl Cardiol 2020:1-9

8. Dahm CN, Cornell RF, Lenihan DJ (2018) Advances in treatment of cardiac amyloid. Curr Treat Options Cardiovasc Med 20:37-37

9. Maurer MS, Schwartz JH, Gundapaneni B et al (2018) Tafamidis treatment for patients with transthyretin amyloid cardiomyopathy. N Engl J Med. 379:1007-1016

10. Bhogal S, Ladia V, Sitwala P et al (2018) Cardiac amyloidosis: An updated review with emphasis on diagnosis and future directions. Curr Probl Cardiol 43:10-34

11. Chacko L, Martone R, Cappelli F, Fontana M (2019) Cardiac amyloidosis: Updates in imaging. Curr Cardiol Rep 21:108-108

12. Perugini E, Guidalotti PL, Salvi F et al (2005) Noninvasive etiologic diagnosis of cardiac amyloidosis using 99mTc-3,3diphosphono-1,2-propanodicarboxylic acid scintigraphy. J Am Coll Cardiol 46:1076-1084

13. Bokhari S, Castaño A, Pozniakoff T, Deslisle S, Latif F, Maurer MS (2013) 99mTc-pyrophosphate scintigraphy for differentiating light-chain cardiac amyloidosis from the transthyretin-related familial and senile cardiac amyloidoses. Circ Cardiovasc Imaging 6:195-201

14. Gillmore JD, Maurer MS, Falk RH et al (2016) Nonbiopsy diagnosis of cardiac transthyretin amyloidosis. Circulation 133:24042412

15. Rapezzi C, Quarta CC, Guidalotti PL et al (2011) Role of 99mTcDPD scintigraphy in diagnosis and prognosis of hereditary transthyretin-related cardiac amyloidosis. JACC Cardiovasc Imaging 4:659-670

16. Quaggin-Smith JA, Wehbe R, Kansal P, Holly TA (2020) Discriminative ability of technetium- $99 \mathrm{~m}$ pyrophosphate imaging for 
ATTR cardiac amyloidosis at a non-amyloid referral center. Abstracts of Original Contributions ASNC2020 The 25th Annual Scientific Session of the American Society of Nuclear Cardiology. J Nucl Cardiol 27:1893

17. Hutt DF, Fontana M, Burniston M et al (2017) Prognostic utility of the Perugini grading of $99 \mathrm{mTc}$-DPD scintigraphy in transthyretin (ATTR) amyloidosis and its relationship with skeletal muscle and soft tissue amyloid. Eur Heart J Cardiovasc Imaging 18:13441350

18. Castano A, Haq M, Narotsky DL et al (2016) Multicenter study of planar technetium $99 \mathrm{~m}$ pyrophosphate cardiac imaging: Predicting survival for patients with ATTR cardiac amyloidosis. JAMA Cardiol 1:880-889
19. Kazi DS, Bellows BK, Baron SJ et al (2020) Cost-effectiveness of tafamidis therapy for transthyretin amyloid cardiomyopathy. Circulation 141:1214-1224

20. Wehbe RM, Kansal P, Holly TA (2020) Cases from a busy nuclear cardiology laboratory: Potential pitfalls in the interpretation of cardiac scintigraphy for ATTR cardiac amyloidosis. J Nucl Cardiol. https://doi.org/10.1007/s12350-020-02094-4

Publisher's Note Springer Nature remains neutral with regard to jurisdictional claims in published maps and institutional affiliations. 